\title{
Formación inicial y carrera docente en América Latina: una mirada global y regional ${ }^{1}$
}

\author{
Initial teacher education and career in Latin America: \\ A global and a regional view
}

Denise Vaillant ${ }^{\mathrm{a}}$ ORCID: 0000-0001-8110-4634

Jesús Mansob ORCID: 0000-0003-1557-3242

Recibido: 6/07/2021 • Aprobado: 10/08/2021

Cómo citar: Vaillant, D., \& Manso, J. Formación inicial y carrera docente en América Latina: una mirada global y regional. Ciencia y Educación, 6(1), 109-118. https://doi.org/10.22206/cyed.2022.v6i1.pp109-118

\section{Resumen}

El presente ensayo teórico surge de una revisión sistemática de la literatura realizada por los autores a partir de las referencias recientes. Tiene por finalidad contribuir a la reflexión y a un diálogo informado acerca de la carrera y la formación de docentes en América Latina a la luz de las tendencias internacionales. Se ofrece, en primer lugar, una revisión sistemática sobre las tendencias y los distintos aspectos que caracterizan las carreras docentes a nivel global. Se parte de la premisa de que la formación docente no puede analizarse de manera aislada, sino que debe estudiarse a partir del escenario en el cual se construye. En una segunda instancia, se centra la mirada en los aspectos más directamente vinculados con la preparación de maestros y profesores en la región. Finalmente, se presentan una serie de sugerencias que permitirían la mejora de la formación en el contexto más amplio de la transformación de las carreras docentes en América Latina.

Palabras clave: formación inicial docente; carrera docente; desarrollo profesional docente; educación superior; América Latina.

\begin{abstract}
This theoretical essay arises from a systematical literature review carried out by the authors based on recent references. Its purpose is to contribute to reflection and informed dialogue on teacher careers and teacher education in Latin America in light of international trends. To this end, first of all, a systematic review of global trends in teacher careers is offered, based on the premise that teacher education cannot be analyzed in isolation, but must be studied from the scenario in which it is constructed. This paper considers aspects related to teaching careers at the international level and the various factors that characterize them. It then focuses on the aspects more directly linked to the preparation of teachers. It concludes with a series of suggestions that would allow for the improvement of training in the broader context of the transformation of teaching careers in Latin America.
\end{abstract}

Keywords: Initial teacher education; teacher career; teacher professional development; higher educationv Latin America.

\footnotetext{
a Universidad ORT, Uruguay. Correo-e: vaillant@ort.edu.uy.

b Universidad Autónoma de Madrid, España. Correo-e: jesus.manso@uam.es

${ }^{1}$ Artículo elaborado en el marco del proyecto \#LobbyingTeachers: Fundamentos teóricos, estructuras políticas y prácticas sociales de las relaciones público-privadas en materia de profesorado en Espańa (Ref. PID2019-104566RA-I00/AEI/10.13039/501100011033). Proyectos de I+D+i del Programa Estatal del Ministerio de Ciencia e Innovación de Espańa.
} 


\section{Introducción}

La temática concerniente a la formación docente constituye una preocupación creciente en la agenda educativa, tanto a nivel internacional como en América Latina. El rol docente adquiere hoy una importancia estratégica en un doble sentido: el vínculo con la creación y la distribución apropiada del conocimiento y la construcción de competencias para la formación continua a lo largo de la vida. Y, en este marco, la preparación del docente cumple un papel clave para el mejoramiento de los sistemas educativos y constituye hoy uno de los desvelos mayores de las políticas educativas (Darling-Hammond, 2017). La reformulación de la formación docente ha sido emprendida en algunos países desde tiempo atrás habida cuenta de las transformaciones en la educación superior y de la necesidad de superar un modelo normalista, ya agotado, en pos de una jerarquización epistemológica, cognitiva, académica y profesional de maestros y profesores (Vaillant \& Marcelo, 2015).

La necesidad de mejora de la formación docente justifica la elaboración de este artículo que presenta una revisión de las tendencias contemporáneas, así como la identificación de opciones posibles para la transformación de las políticas actuales. La evidencia que se presenta fue compilada en una revisión sistemática de la literatura a cargo de los autores. En concreto, nos hemos basado en las bases de datos ERIC y SCOPUS y en antecedentes y referencias fundamentadas que figuran en trabajos previos ya publicados (Manso \& Garrido, 2021; Vaillant, 2021; Vaillant \& Manso, 2013). Para delimitar el corpus de estudios que conforma la revisión de la literatura, se adoptó el marco metodológico diseñado por Wolfswinkel, Furtmueller \& Wilderom (2013), basado en las siguientes etapas: (i) delimitación y búsqueda; (ii) selección e identificación de temas emergentes; (iii) interpretación de resultados; y (iv) elaboración de conclusiones. Conforme a las etapas realizadas, la estructura del artículo se organiza con una primera parte en la que se abordan los factores intervinientes en la configuración de las carreras docentes a la luz de las tendencias internacionales. En otro apartado se examinan diversas estrategias para implementar cambios en la formación docente desde una perspectiva global y con enfoque de la región. Por último, se comparten reflexiones y recomendaciones para la toma de decisiones, con especial énfasis en América Latina.

\section{Tendencias globales en materia de carreras docentes}

La atracción y retención de personal competente en una profesión que ha perdido su prestigio social y su estatus en las últimas décadas ha sido una preocupación constante de responsables educativos, técnicos, educadores e investigadores (González Castro, 2020). Para indagar en posibles caminos de solución, se han llevado adelante estudios internacionales y regionales que han identificado los obstáculos, las insuficiencias en el diseño e implementación y las reformulaciones necesarias en las políticas docentes (Clark \& Newberry, 2019; Mourshed, Chijioke \& Barber, 2010; OCDE, 2018; 2019; Sharpes, 2020).

Informes como el de la UNESCO (2014) o el de Bruns \& Luque (2014) subrayan que la problemática educativa se asocia en buena medida con la necesidad de contar con políticas docentes sistémicas y de largo alcance. Coinciden en la idea de que hay que llevar adelante acciones tanto a nivel macro del sistema educativo (condiciones laborales y carrera docente) como a nivel de los docentes (incorporación de capacidades y competencias para mejores logros educativos de los alumnos).

El nivel macro y la carrera docente aluden a la normativa que regula el ejercicio de la profesión y, en particular, aspectos tales como el ingreso, la movilidad, el desarrollo, el ascenso y el retiro de las personas que ejercen la profesión docente (Vaillant \& Marcelo, 2015; Valle \& Manso, 2017).

No obstante, la conceptualización de la carrera docente ha sufrido muchos cambios. En décadas pasadas, el significado asociado a la carrera se relacionaba con algo positivo, lineal y predecible con relación a las jerarquías establecidas dentro de una organización (Valle \& Manso, 2017). En la actualidad, la carrera docente se define en torno al aprendizaje y el desarrollo profesional. Se espera que la carrera docente 
se adapte a los cambios educativos, al desarrollo de las capacidades y competencias necesarias para avanzar en la profesión.

Todavía hoy siguen siendo muchos los países que ofrecen un sistema de carrera profesional "plana” (Eurydice, 2018); es decir, que no contemplan distintos niveles que incentiven y acompañen adecuadamente una evolución progresiva de los desempeńos docentes. De esta manera, se darían situaciones en las que un aspirante recién ingresado a la profesión pudiera no recibir más formación permanente o no tener ningún apoyo de evaluación que le permita seguir mejorando su práctica profesional. Por el contrario, la evidencia apunta a la necesidad de implementar un sistema de carrera "multi-nivel" (Eurydice, 2018) que mejore la satisfacción con el trabajo y la confianza organizacional (Li, Pérez-Díaz, Mao \& Petrides, 2018).

Así, las tendencias a nivel internacional muestran una enorme diversidad en los mecanismos referidos a las diversas etapas de la carrera y los criterios de selección de aspirantes (Eurydice, 2018). De entre todos esos mecanismos, se destacan aquellos que hacen referencia a: (i) los marcos normativos, (ii) las titulaciones o credenciales y los criterios de selección para ingresar en la profesión y (iii) la evaluación de los desempeños docentes.

La diversidad constatada en torno a las normas que regulan la profesión se vincula estrechamente con la capacidad de atracción y retención de los docentes en los sistemas educativos; aspecto, por otro lado, que sabemos tiene una enorme relevancia a la hora de definir el prestigio social y la identidad profesional de los docentes (Manso \& Valle, 2019). Es el marco normativo el que garantiza que aquellos que ingresan a la profesión lo hacen con las competencias para el ejercicio de una docencia de calidad (Vaillant, 2019). Es la normativa de cada país el referencial para otorgar el título docente y habilitar al ejercicio de la profesión. Esa habilitación varía según los contextos nacionales y puede estar a cargo del Estado nacional, (tal es el caso de Francia) o bien de Estados federales, provincias o municipios (por ejemplo, Estados Unidos). En algunos países la habilitación está a cargo de las instituciones formadoras (Nueva Zelanda).
Los criterios de selección para ingresar en la profesión docente difieren según el sistema educativo. En algunos casos, los requerimientos refieren al nivel de estudios previos y a pruebas que permitan demostrar la idoneidad para ejercer la docencia. Así, por ejemplo, en Australia, Finlandia y Singapur los egresados de profesorados de educación media deben aprobar exámenes nacionales. En países como Dinamarca, Finlandia, Corea, Escocia y Singapur se realizan entrevistas para valorar la idoneidad de los postulantes para ingresar a la profesión (Kim, Jörg \& Klassen, 2019; OCDE, 2019).

En Australia y Canadá, además de la titulación docente, se exige una habilitación a nivel de una especialización que acredite que los docentes están plenamente cualificados (OCDE, 2018). En este sentido, en el último informe TALIS (Teaching and Learning International Survey) de la OCDE (2019) se indica que, en los países pertenecientes a la organización, la mitad de los docentes cuenta con un único título de grado, mientras que un $44 \%$ posee una titulación especializada adicional a nivel de maestría.

Entre los casos de interés en materia de criterios de selección de docentes a nivel internacional, puede mencionarse el caso de Inglaterra, que ofrece modalidades alternativas para ingresar a la docencia. Las reformas iniciadas en la década de los ochenta habilitaron a asociaciones de centros educativos a transformarse en instituciones formadoras. Mediante ese mecanismo fueron creadas instituciones estatales que regularon el sistema y otorgaron el Qualified Teacher Status (Furlong, 2013). En este contexto surgieron nuevos organismos para definir estándares y administrar los programas de formación, entre otras tareas.

Además, también a nivel internacional, los cambios en materia de carrera docente se vinculan claramente con la temática de la evaluación del desempeño. Una revisión de la literatura acerca de la evaluación del desempeño docente muestra que es necesario articular la carrera con la formación (Tarhan, Karaman, Lauri \& Aerila, 2019). Este es un aspecto clave a la hora de planificar nuevas ofertas y apoyo a la labor educativa.

Existe una gran diversidad en materia de evaluación del desempeño docente que responde a diversos 
enfoques (OCDE, 2018; 2019). La conceptualización del proceso de evaluación registra diferencias importantes: evaluación al finalizar un período de prueba; evaluación vinculada al desempeño (por ej. para la promoción) y evaluación asociada al sistema de incentivos. Y también hay apreciables distinciones a realizar a la hora de definir la finalidad o las consecuencias de esta evaluación. Según datos de Eurydice (2018), en algunas ocasiones estos mecanismos se focalizan esencialmente en dar retroalimentación destinada a la mejora sucesiva (como en Lituania o la República Checa); sin embargo, en la mayoría de los casos está asociada exclusivamente a la mejora de las condiciones profesionales y salariales.

Entre los países o las provincias más citados en la literatura, figura el caso de Ontario, Canadá, donde es posible distinguir dos niveles en los sistemas de evaluación. El primero se vincula con los nuevos docentes y un programa de inducción obligatorio que dura un año y que se integra por la orientación y el mentorazgo a cargo de profesores experimentados (Glassford \& Salinitri, 2007) ${ }^{\text {a }}$ El segundo nivel del sistema de evaluación en Ontario se asocia a los profesores experimentados, que deben contar con un plan de desarrollo profesional a partir del cual es valorado su desempeño por parte del equipo directivo de su centro educativo. Tanto los profesores nuevos como los experimentados con calificaciones insatisfactorias reciben pautas para mejorar su desempeño en el aula (OCDE, 2019).

Un país de interés es Singapur, donde los docentes son evaluados después de tres años de labor. Esa evaluación permite determinar cuál será su rol: profesor maestro, especialista en currículum, investigador o directivo escolar. En el caso de los directivos, estos reciben una formación adicional para el ejercicio de su rol (Schleicher, 2014).

En Finlandia, la evaluación docente se caracteriza por ser grupal, reflexiva y participativa. Tiene como principal fin crear comunidades de aprendizaje profesional entre docentes y directivos escolares. En este sentido, la evaluación no solo tiene que ver con el desempeño del docente, sino que además se centra en conocer las percepciones de maestros y profesores sobre su trabajo, las condiciones laborales y su desarrollo profesional. La evaluación docente tiene lugar durante las conversaciones entre docentes y directivos, junto a colegas que dictan la misma materia o el mismo grado escolar (Tarhan, Karaman, Lauri \& Aerila, 2019).

\section{Los procesos de formación docente y las tendencias internacionales}

La configuración concreta del proceso formativo, sus condiciones y requisitos, su duración y nivel se han transformado en las últimas décadas (Valle \& Manso, 2017). Hoy la formación del docente es objeto de renovado interés. Como en el apartado anterior, en los párrafos que siguen se ofrece una aproximación sobre la formación docente a la luz de diferentes casos internacionales de interés para América Latina.

Es sabida la importancia de atraer a estudiantes con buenos rendimientos académicos para cursar estudios de docencia, lo que requiere de una estructura escolar y social que otorgue alta valoración social a la profesión. El estudio de Podolsky, Kini, Darling-Hammond \& Bishop (2019) confirma que la permanencia y la culminación de estudios de formación docente están altamente relacionadas con la preparación que los candidatos tengan al ingresar.

Junto con la selección de acceso a la formación inicial, el propio periodo formativo previo al ejercicio profesional constituye un elemento esencial en el desarrollo profesional docente (Valle \& Manso, 2017). Resulta evidente la necesidad de entender la formación como un sistema articulado a lo largo de la vida profesional que, de manera coherente y sustentable, genere un continuo de aprendizaje desde la formación inicial, la formación en servicio y la carrera docente (Vaillant \& Marcelo, 2015).

\footnotetext{
${ }^{a}$ Sobre los programas de inducción a la profesión, conviene indicar que también en la región (América Latina) se han comenzado a poner en marcha algunas acciones de interés. Para profundizar en ellos, se considera oportuno revisar el recién artículo de Vaillant (2021) sobre la inserción del profesorado novel en la región.
} 
A nivel internacional, la preparación de los docentes ocurre en universidades o en instituciones de nivel terciario. En algunos países, como en Estados Unidos, Canadá, Australia, Holanda o Inglaterra, la formación se desarrolla en las universidades. En otros casos, como en Singapur, el proceso formativo se lleva a cabo en una sola institución denominada National Institute of Education (Vaillant, 2018).

A nivel de la malla curricular, la mayoría de los programas articulan conocimientos disciplinares, pedagógicos y prácticas docentes, y son impartidos por un profesorado específico. La duración oscila entre tres y seis ańos, lo que denota una alta disparidad que se refleja luego en los contenidos aprendidos por los profesores en formación (Liu \& Liao, 2019). La tendencia actual es una duración de entre 4 y 5 ańos, que se ha ampliado en algunos países por la necesidad de obtención del título de Magíster para poder ejercer la docencia (Flores, 2016).

Con independencia de la duración de los estudios, el incremento de exigencia para la obtención del título de docente tiene consecuencias positivas y negativas (Loughran \& Hamilton, 2016). En algunos casos, la exigencia de títulos universitarios de grado y posgrado para el ejercicio docente ha acentuado el divorcio entre la teoría y la práctica, entre el conocimiento proposicional y el conocimiento práctico, entre la racionalidad técnica y la epistemología de la práctica (Prats, 2016).

Esa creciente separación entre formación docente y escuela ha resultado en que muchos países implementen otras vías para la certificación y acreditación docente, con menor grado de presencia e influencia de las universidades. En este sentido, en países como Estados Unidos, desde hace más de cuatro décadas, los programas de formación alternativa de docentes preparan a un tercio de los docentes en ese país (Zeichner, 2016).

Los programas alternativos de formación docente tienen, en general, una duración de uno a dos años, y puede accederse a ellos luego de haber obtenido un título de grado universitario. Los candidatos certificados mediante estos programas reciben una formación centrada en metodología didáctica y gestión del aula, ya que el conocimiento disciplinar, acreditado mediante el título de grado, es un requisito para el ingreso. Según el estado, pueden tener o no un vínculo directo con las universidades (Adcock \& Mahlios, 2005). Los programas son flexibles en sus horarios y formas de cursarlos e incorporan un componente de práctica docente y mentorazgo.

Existen numerosas y diversas reformas a nivel internacional en materia de formación de docentes, entre las que se destacan los casos de Australia, Alemania y Singapur. Se trata de transformaciones que se inscriben en el contexto más amplio de la mejora de la profesión docente, que constituye el foco del presente artículo (Vaillant, 2018).

En Australia, los maestros y profesores se forman en unas 40 universidades, lo que ha otorgado gran relevancia a los procesos de acreditación debido a la gran cantidad y diversidad de las instituciones (Vaillant, 2018). El sistema nacional de regulación de la profesión docente está a cargo de una agencia denominada Australian Institute for Teaching and School Leadership (AITSL), uno de cuyos principales objetivos es el de desarrollar y mantener rigurosos estándares profesionales para docentes (Darling-Hammond, 2017). La diversidad de los procesos formativos en las universidades es grande, pero al mismo tiempo existe una marcada regulación basada en un conjunto de criterios nacionales (Darling-Hammond, 2017).

Entre los Estados australianos, se destaca el proceso de acreditación llevado adelante por el Estado de Victoria, administrado por el Victorian Institute of Teaching (VIT) desde el año 2004. Para acreditarse, las instituciones formadoras deben proveer información al VIT a los efectos de que este evalúe en qué medida estas cumplen con los requisitos establecidos (Mayer, 2014). En cuanto a los docentes, estos deben solicitar una autorización provisoria para ejercer la docencia al término de sus estudios universitarios. Para postular a la siguiente autorización válida por dos años, los docentes deben haber impartido clases por un mínimo de 80 días. El director de la escuela donde trabajó el docente debe entregar evidencia del cumplimiento de estándares en tres ámbitos: conocimiento profesional, habilidades prácticas y compromiso profesional. 
En Alemania, la formación docente se organiza en función de los diversos niveles educativos. Existen profesores de educación primaria, profesores de ciclo básico, profesores de bachillerato, maestros de escuelas vocacionales y maestros para estudiantes con discapacidad mental o física. Por tratarse de un país federal, son los 16 estados (Länder) los que tienen la responsabilidad de la formación docente. La Conferencia Permanente de los Ministros de Educación y Asuntos Culturales es la que brinda un marco común y estándares para la formación docente. Para ser maestro o profesor, de los niveles previamente mencionados, los candidatos deben haber finalizado los 13 años de escolarización obligatoria. Luego del ingreso, se desarrolla la primera fase de formación, en una universidad o un colegio de arte o música, y tiene una duración de 3-4 años (para los candidatos de enseñanza primaria) a 4-5 o incluso 6 años (para los candidatos de enseñanza secundaria). Los cursos impartidos durante esta etapa incluyen el estudio de al menos dos asignaturas, áreas temáticas o grupos de asignaturas. También se deben cursar materias referidas a teoría educativa y psicología, cursos optativos y trabajos prácticos en las escuelas (Terhart, 2019). Una vez culminada la primera fase, los candidatos inician una segunda fase de formación práctica durante dos años. Durante esta segunda fase el candidato recibe un salario. Al culminar, debe presentar un trabajo monográfico vinculado a la didáctica de una de las asignaturas estudiadas en la universidad, un examen de enseñanza práctica en cada asignatura, exámenes orales sobre cuestiones didácticas y metodológicas, teoría educativa y temas legales de la educación. Luego de la culminación de sus estudios, los docentes son considerados funcionarios públicos y, por tanto, son empleados estatales (Terhart, 2019).

Por su parte, Singapur es otro de los países que se destaca por la estructuración de la propuesta de formación docente. En ese país los profesionales de la educación son prestigiosos y tienen un salario similar al de un ingeniero. Los docentes reciben complementos salariales en función del desempeño general del centro educativo, de los resultados de los alumnos y de la antigüedad (Vaillant, 2018). Existe un único centro que imparte formación docente. Se trata del National Institute of Education (NIE), el cual depende del Ministerio de Educación. El NIE establece las regulaciones para formar a los futuros docentes en base al currículo único nacional. Los candidatos aceptados en el NIE son considerados como funcionarios públicos y reciben una remuneración durante su formación (Goodwin, 2012). Para su ingreso al NIE, los candidatos deben sortear con éxito una entrevista y una experiencia de inmersión en un centro educativo donde se observa su motivación y vocación (Goodwin, 2012). Todos los docentes graduados del NIE tienen empleo garantizado. Tras el ejercicio de la profesión durante tres años, se evalúa a los docentes anualmente para determinar los aumentos salariales (Schleicher, 2014).

A la luz de estos casos, resulta pertinente volver la mirada a América Latina y analizar los desafíos que hoy presenta la formación docente en la región. Asumiendo la enorme diversidad de países e instituciones, hemos identificado algunos aspectos críticos que suponen un reto para toda la región.

\section{Nudos problemáticos y desafíos de la formación docente en América Latina}

Uno de los principales desafíos en América Latina es la mejora de la formación docente, con particular énfasis en el profesorado de educación secundaria. Históricamente, las estrategias planteadas tienden a no cuestionar los principios de estos modelos tradicionales. De hacerlo, las iniciativas innovadoras tienen lugar en contextos específicos y no de masividad.

Entre los retos de la formación inicial en América Latina aparece la estructura organizativa, que presenta una fuerte dicotomía vinculada a las prioridades de los países. La formación del docente se imparte en algunos casos en universidades a través de facultades de educación. También existen universidades pedagógicas y los institutos de formación docente de nivel terciario no universitario (Vaillant, 2019).

Un segundo desafío es la selección de los candidatos a docentes. Con frecuencia, los candidatos deben evidenciar un buen desempeño en matemática y lengua, además de competencias interpersonales y una 
fuerte motivación para enseñar y aprender (Vaillant, 2019). Más recientemente, algunos países latinoamericanos han incluido entre los mecanismos de selección pruebas asociadas a la personalidad de candidatos para evitar eventuales efectos psicológicos negativos sobre sus alumnos (Vaillant, 2019).

El tercer desafío se asocia a los lineamientos curriculares debido a que, en buena parte de los países latinoamericanos, las mallas curriculares se caracterizan por una acumulación de saberes conceptuales en el ámbito disciplinar y pedagógico (Vaillant, 2018). Así, la formación de los profesores de secundaria suele ser fragmentaria y dispersa, con poco énfasis en la enseñanza en el contexto real de aula (Gatti \& Barreto, 2009; Calvo, Rendón \& Rojas, 2004; Ávalos \& Matus, 2010). Algunos países tienen marcos referenciales para las propuestas curriculares de formación docente, como en el caso de Chile, Perú y Ecuador. Otros, como Brasil, tienen orientaciones de carácter bastante general, sin detallar contenidos específicos que son definidos por las instituciones formadoras (Gatti \& Davis, 2016).

La temática referida a los formadores de docentes es el cuarto desafío identificado. La evidencia es escasa en América Latina acerca de la mayor o menor incidencia que tienen los formadores en los candidatos a la docencia (Ávalos \& Matus, 2010). Los datos del estudio IEA TEDS-M señalan que los equipos de formadores tienen escasos conocimientos acerca de los centros educativos en los cuales se desempeñarán los futuros docentes a los que preparan (Ávalos \& Matus, 2010).

Un quinto desafío se vincula con la práctica docente, la cual registra la misma heterogeneidad detectada para la estructura de la formación docente. Cuando la preparación del profesorado se desarrolla en institutos de formación docente dependientes de los Ministerios de Educación, existe una fuerte presencia de las prácticas, pero poca reflexión teórica (Louzano \& Moriconi, 2014). Inversamente, cuando la formación es universitaria, la reflexión y el análisis están presentes, pero las experiencias prácticas son escasas (Vaillant, 2018). Una tendencia constatada en los últimos años es la presencia de programas a distancia o que se realizan solamente los fines de semana, en los cuales la práctica docente es inexistente o se desarrolla de manera precaria (Vaillant, 2018).

Finalmente, identificamos otro desafío vinculado con los procesos de regulación y acreditación. La temática tiene extrema vigencia debido a que, en muchos países latinoamericanos, existe una enorme proliferación y dispersión de la formación docente. Muchas instituciones tienen pequeñas dimensiones y falta el equipamiento adecuado (bibliotecas, computadoras, laboratorios) para asegurar una formación de calidad y una equitativa distribución geográfica de la oferta (UNESCO, 2014).

\section{Hacia dónde avanzar para la mejora de los procesos de formación}

Para pensar las opciones de políticas y estrategias a impulsar en el futuro en materia de formación docente, es necesaria una mirada desde lejos y a lo lejos. El proceso de transformación de la formación docente debería encararse de forma sistémica, abarcando simultáneamente una batería de estrategias y programas que permitan revertir los graves problemas de eficiencia interna y externa del sistema actual.

A modo de decálogo, y con base en las tendencias internacionales y regionales que hemos identificado en apartados previos, es que formulamos una serie de sugerencias que podrían permitir la mejora de la formación:

- promover políticas para atraer los mejores candidatos en la docencia;

- seleccionar formadores con capacidades, competencias y experiencia escolar relevante;

- establecer mecanismos de articulación entre las instituciones formadoras y los centros educativos donde se realizan las prácticas profesionales;

- gestionar con eficiencia las instituciones de formación a través de procedimientos de acreditación;

- identificar sistemas de competencias o estándares que sirvan para orientar la formulación de contenidos curriculares y la evaluación de los logros de aprendizaje de los futuros docentes; 
- impulsar la mejora de la calidad de los programas de formación según lo requerido en los niveles educacionales para los que se prepara;

- promover el desarrollo continuo del docente centrado en su desempeńo en el aula;

- estimular la colaboración entre colegas para aprender unos de otros;

- implementar programas efectivos de iniciación de los docentes nóveles.

La última sugerencia referida a los programas de iniciación a la docencia es quizás un terreno fértil para poder avanzar a corto plazo. Son numerosos los estudios que recientemente insisten en la relevancia de cuidar la iniciación de los docentes nóveles en el puesto de trabajo (Bedacarratx, 2021; Manso \& Garrido, 2021). En la región, Vaillant (2021) nos recuerda que son pocos los países con programas de iniciación profesional. Entre estos se destacan los de Chile y, más recientemente, el programa puesto en marcha en República Dominicana (López \& Marcelo, 2021).

Valle \& Manso (2017) señalan el relevante papel que tiene la inserción para integrar la formación inicial y la continua como manera de acelerar las mejoras en el desarrollo profesional. La inserción docente contribuye a mejorar la formación inicial (ya que tiene que tener en cuenta la posterior inserción de sus egresados en el programa de inducción), favorece una cultura de centro colegiada y una evaluación docente centrada en la mejora sucesiva, aumenta la colaboración y el aprendizaje cooperativo entre docentes e incorpora una carrera docente en la que existen distintos estadios sucesivos (multinivel) por los que los docentes van pasando a medida que mejoran su práctica. Por último, ayuda a resolver unos de los principales problemas identificados en este artículo: la difícil vinculación entre la teoría y la práctica en los diferentes programas de formación docente.

Todo esto nos orienta hacia aquel profesional reflexivo que tiene las herramientas teórico-prácticas oportunas para poder aplicar con criterio pedagógico las acciones oportunas en los contextos concretos en los que se desarrolla su acción educativa. El acercamiento a un docente profesionalizado y reflexivo (en suce- siva formación y mejora) tendrá repercusiones directas sobre su propia imagen (identidad profesional) y sobre su valoración externa (prestigio social).

\section{Referencias}

Adcock, P., \& Mahlios, M. (2005). Nontraditional alternative teacher certification programs: Their purpose, design and participants. Essays in Education, 15.

Ávalos, B. \& Matus C. (2010). La Formación Inicial Docente en Chile desde una óptica internacional. Ministerio de Educación (Chile).

Bruns, B. \& Luque, J. (2014). Docentes excelentes: cómo mejorar el aprendizaje en América Latina y el Caribe. Banco Mundial.

Bedacarratx, V. (2021). Sujetos y afectos en la inserción al mundo profesional de la docencia. Ciencia y Educación, 5(2), 7-24. https://doi. org/10.22206/cyed.2021.v5i2.pp7-24

Calvo, G., Rendón, D. B. \& Rojas, L. I. (2004). Un diagnóstico de la formación docente en Colombia. Revista Colombiana de Educación, 47. https://doi.org/10.17227/01203916.5519

Clark, S., \& Newberry, M. (2019). Are we building preservice teacher self-efficacy? A large-scale study examining teacher education experiences. Asia-Pacific Journal of Teacher Education, 47(1), 32-47. https://doi.org/10.1080/1359866X.2018. 1497772

Cuenca, R. (2015). Las carreras docentes en América Latina: la acción meritocrática para el desarrollo profesional. UNESCO-OREALC.

Darling-Hammond, L. (2017) Teacher education around the world: What can we learn from international practice? European Journal of Teacher Education, 40(3), 291-309. https://doi.org/10. 1080/02619768.2017.1315399

Eurydice. (2018). La profesión docente en Europa: Acceso, progresión y apoyo. Informe de Eurydice. Oficina de Publicaciones de la Unión Europea. Flores, A. (2016). Teacher Education Currículum. In J. Loughran \& M. Hamilton (Eds.), International Handbook of Teacher Education (pp. 187-230). Springer. 
Furlong, J. (2013). Education-An Anatomy of the Discipline: Rescuing the university project? Routledge.

Gatti, B.A., \& Barretto, E.S. (2009). Professores do Brasil: impasses e desafios. UNESCO.

Gatti, B. A. \& Davis, C. (2016). El papel de las instituciones de educación superior en la formación de profesores en América Latina. Banco Mundial.

Glassford, L., \& Salinitri, G. (2007). Designing a successful new teacher induction program: An assessment of the Ontario experience, 2003-2006. Canadian Journal of Educational Administration and Policy, 60.

González Castro C. (2020). Desvalorización de la profesión docente en Chile: capitalismo tardío y profesorado. Ciencia y Educación, 4(3), 157-166. https://doi.org/10.22206/cyed.2020.v4i3. pp157-166

Goodwin, A.L. (2012). Quality teachers, Singapore style. In L. Darling-Hammond \& A. Liberman (eds.), Teacher Education around the World. Routledge.

Kim, L., Jörg, V., \& Klassen, R. M. (2019). A MetaAnalysis of the Effects of Teacher Personality on Teacher Effectiveness and Burnout. Educational psychology review, 31(1), 163-195. https://doi. org/10.1007/s10648-018-9458-2

Li, M., Pérez-Díaz, P.A., Mao, Y., \& Petrides, K.V. (2018). A multilevel model of teachers' job performance: Understanding the effects of trait emotional intelligence, job satisfaction, and organizational trust. Frontiers in Psychology, 9, 2420. https://doi.org/10.3389/fpsyg.2018.02420

Liu, Y., \& Liao, W. (2019). Professional development and teacher efficacy: evidence from the 2013 TALIS. School Effectiveness and School Improvement, 30(4), 487-509. https://doi.org/10.108 $0 / 09243453.2019 .1612454$

López, A. \& Marcelo, C. (2021). La inducción a la enseñanza en la República Dominicana: El Programa Nacional de Inducción. Ciencia y Educación, 5(2), 95-120. https://doi.org/10.22206/cyed.2021. v5i2.pp95-120

Loughran, J., \& Hamilton, M. (2016). International Handbook of Teacher Education. Springer.
Louzano, P. \& Moriconi, G. (2014). Visión de la docencia y características de los sistemas de formación docente. En UNESCO/ OREALC (ed.), Temas criticos para formular nuevas politicas docentes en América Latina y el Caribe: el debate actual (pp. 10-52). UNESCO-OREALC.

Manso, J. \& Valle, J. M. (2019). La iniciación del profesional docente: planteamientos sobre la formación inicial y los programas de inducción. En Comunidad de Madrid, La mejora de la profesión docente en la Comunidad de Madrid (pp. 84-99). Comunidad de Madrid.

Manso, J. \& Garrido, R. (2021). Los que empiezan: el reto pendiente de acompańar a docentes noveles. Profesorado. Revista de Curriculum y Formación de Profesorado, 25(2), 145-163. https://doi. org/10.30827/profesorado.v25i2.18441

Mayer, D. (2014). Forty years of teacher education in Australia: 1974-2014. Journal of Education for Teaching, 40(5), 461-473. http://dx.doi.org/1 0.1080/02607476.2014.956536

Mourshed, M., Chijioke, C., \& Barber, M. (2010). How the world's most improved school systems keep getting better. McKinsey.

OCDE. (2018). Effective Teacher Policies Insights from PISA. OECD Publishing. http://dx.doi. org/10.1787/9789264301603-en

OCDE. (2019). TALIS 2018 Results (Volume I): Teachers and school leaders as lifelong learners. OECD Publishing.

Podolsky, A., Kini, T., Darling-Hammond, L., \& Bishop, J. (2019). Strategies for attracting and retaining educators: What does the evidence say? Education policy analysis archives, 27(38). http://dx.doi.org/10.14507/epaa.27.3722

Prats, E. (2016). La formación inicial docente entre profesionalismo y vías alternativas: mirada internacional. Bordón. Revista de pedagogía, 68(2), 19-33. https://doi.org/10.13042/Bordon. 2016.68202

Schleicher, A. (2014). Equity, Excellence and Inclusiveness in Education: Policy Lessons from Around the World, International Summit on the Teaching Profession. OECD Publishing. https://doi.org/ 10.1787/9789264214033-en 
Sharpes, D.K. (2020). Education and the US Government. Routledge.

Tarhan, H., Karaman, A., Lauri, K., \& Aerila, J. A. (2019). Understanding teacher evaluation in Finland: A professional development framework. Australian Journal of Teacher Education, 44(4), 33. https://doi.org/10.14221/AJTE. 2018V44N4.3

Terhart, E. (2019). Critical Overview of Teacher Education in Germany. Oxford Research Encyclopedia of Education. https://doi.org/10.1093/ acrefore/9780190264093.013.377

UNESCO. (2014). Antecedentes y Criterios para la Elaboración de Politicas Docentes en América Latina y el Caribe. CEPPE y UNESCO.

Vaillant, D. (2013). Formación inicial del profesorado en América Latina: dilemas centrales y perspectivas. Revista Española de Educación Comparada, 22, 185-206.

Vaillant, D., \& Manso, J. (2013). Teacher education programs: learning from worldwide inspiring experiences. Journal of Supranational Policies of Education 1, 94-115.

Vaillant, D. (2018). Estudio exploratorio sobre los modelos organizacionales y pedagógicos de instituciones dedicadas a la formación docente inicial: un análisis en clave comparada. Informe final. INFOD e IIPE-UNESCO.
Vaillant, D. (2019). Formación inicial del profesorado de educación secundaria en América Latina- dilemas y desafíos. Profesorado. Revista de Curriculum y Formación de Profesorado, 23(3), 35-52. https://doi.org/10.30827/profesorado.v23i3.9516

Vaillant, D. (2021). La inserción del profesorado novel en América Latina: Hacia la integralidad de las políticas. Profesorado. Revista de Curriculum y Formación de Profesorado, 25(2), 79-97. https:// doi.org/10.30827/profesorado.v25i2.18442

Vaillant, D. \& Marcelo, C (2015). El A, B, C, D de la formación docente. Narcea.

Valle, J., \& Manso, J. (2017). Lifelong Teacher Education: ser docente a lo largo de la vida. CDL-Pigmalion.

Wolfswinkel, J., Furtmueller, E., \& Wilderom, C. (2013). Using grounded theory as a method for rigorously reviewing literature. European Journal of Information Systems, 22(1), 45-55.

Zeichner, K. (2016). Independent Teacher Education Programs: Apocryphal Claims, Illusory Evidence. National Education Policy Center. 